\title{
Diseño, validación y aplicación de un cuestionario para medir la influencia de factores exógenos sobre la eficacia del aprendizaje invertido
}

\author{
Arturo FUENTES CABRERA ${ }^{1}$, Jesús LÓPEZ BELMONTE ${ }^{2}$, \\ María Elena PARRA GONZÁLEZ ${ }^{1}$ y María Belén MORALES CEVALLOS ${ }^{3}$
}

\author{
${ }^{1}$ Universidad de Granada \\ ${ }^{2}$ Universidad Internacional de Valencia \\ ${ }^{3}$ Universidad Católica Santiago de Guayaquil
}

(Recibido el 6 de Marzo de 2019, Aceptado el 26 de Agosto de 2019)

\begin{abstract}
RESUMEN: Los progresos acaecidos en materia tecnológica han provocado la aparición de nuevos modelos de aprendizaje en el mundo de la educación. Entre estos modelos se encuentra el aprendizaje invertido. El objetivo de este estudio pasa por diseñar y validar un instrumento para valorar la eficacia del aprendizaje invertido, así como por conocer la incidencia de determinadas variables sociales y exógenas en su aplicación. Para ello se ha seguido un método cuantitativo a través de un diseño experimental de corte longitudinal, descriptivo y correlacional, en una muestra de 360 discentes universitarios. Para la recogida de datos se ha empleado el cuestionario generado tras el proceso de validación. Los resultados obtenidos han permitido concluir que valores adecuados en el contexto familiar, motivación, autoestima y autonomía, inciden favorablemente en la eficacia del enfoque innovador. Asimismo, la edad y el desempeño laboral de los estudiantes también resultan influyentes. Del mismo modo, se ha podido determinar qué factores externos ejercen mayor incidencia sobre cada aspecto constitutivo de la eficacia del aprendizaje invertido.
\end{abstract}

Palabras clave: cuestionario; tecnología de la educación; metodología; innovación pedagógica; condiciones de aprendizaje.

\section{Design, validation and application of a questionnaire to measure the influence} of exogenous factors on the effectiveness of flipped learning

\begin{abstract}
The progress made in technology has led to the emergence of new learning models in the world of education. Among these models is flipped learning. The objective of this study is to design and validate an instrument to assess the effectiveness of flipped learning, as well as to know the incidence of certain social and exogenous variables in its application. For this, a quantitative method has been followed through an experimental design of longitudinal, descriptive and correlational cut, in a sample of 360 university students. For the collection of data, the questionnaire generated after the validation process was used. The results obtained have allowed us to conclude that adequate values in the family context, motivation, self-esteem and autonomy, favorably affect the effectiveness of the
\end{abstract}


innovative approach. Also, the age and work performance of students are also influential. In the same way it has been possible to determine which external factors exert greater incidence on each constitutive aspect of the effectiveness of flipped learning.

Keywords: questionnaire; educational technology; methodology; educational innovation; learning conditions.

Correspondencia: Jesús López Belmonte. Cortadura del Valle sn. CP: 51001. Ceuta (España). E-mail: jesus.lopezb@,campusviu.es

\section{Introducción}

La sociedad actual se ha visto sometida a diversos cambios en los últimos años que han afectado de manera crucial a la población y a aspectos de carácter económico, cultural o tecnológico, incidiendo directamente en unas transformaciones de diversa índole en la educación de nuestros días (Rotellar y Cain, 2016). Sobre todo, este cambio ha venido acompañado de una nueva y diferente manera de acceder a la información (López y Bernal, 2019), provocando una transformación en los procesos de enseñanza-aprendizaje, apoyados masivamente por el progreso de las tecnologías de la información y la comunicación (TIC) y en la aparición de nuevos espacios de aprendizaje de índole digital (McCarthy, 2016).

La continua incidencia de las TIC ha ocasionado que los expertos aboguen por la inclusión de estas tecnologías de uso didáctico en los centros educativos (Area, Hernández y Sosa, 2016), así como impulsar medidas de formación del profesorado en materia de innovación tecnológica aplicada a la educación (Fernández, Fernández y Rodríguez, 2018). Todo ello está centrado en la búsqueda de la optimización de los procesos educativos que tienen como protagonista - en la actualidad - a la figura del alumnado como principal agente del proceso de aprendizaje (Jovanović, Gašević, Dawson, Pardo y Mirriahi, 2017).

Con toda la transformación tecnopedagógica cabe destacar la aparición de gran cantidad de metodologías de enseñanza y aprendizaje. De entre todos los nuevos enfoques metodológicos, Sánchez (2017), Seery (2015), Zainuddin, Habiburrahim, Muluk y Keumala (2019) hacen mención a uno que ha adquirido un gran protagonismo en los últimos años: el aprendizaje invertido o flipped learning. Este enfoque pedagógico innovador se ha convertido en un referente esencial debido a los altos índices de éxito y efectividad que ha venido demostrando en estudios anteriores (He, Holton, Farkas y Warschauer, 2016; Pereira, Fillol y Moura, 2019).

El aprendizaje invertido es definido como un enfoque pedagógico mixto (Lee, Lim y Kim, 2017) en el que los roles tradicionales del aula se encuentran alterados (Mortensen y Nicholson, 2015), consiguiendo que sea el discente quien comience su proceso de aprendizaje en un contexto distinto al tradicional y pueda continuarlo y reforzarlo en el horario lectivo (Llong, Cummins y Waugh, 2017). Así, se produce un aprendizaje basado fundamentalmente en la utilización de recursos tecnológicos (Froehlich, 2018). Del mismo modo, este modelo innovador permite una mayor flexibilidad, tanto del tiempo como del espacio, en comparación con otros enfoques pedagógicos (Boelens, Voet y Wever, 2018).

Un reciente estudio realizado por El Miedany (2019) asegura que el aprendizaje invertido logra una mayor profundización en los contenidos, gracias a que el tiempo destinado en clase es mucho más productivo. Asimismo, MacLeod, Yang, Zhu y Shi (2017) exponen que este enfoque 
permite un mayor grado de colaboración entre el alumnado, favoreciendo la socialización y motivación por el aprendizaje (Shih y Tsai, 2017; Tse, Choi y Tang, 2019).

Del mismo modo, este modelo invertido de aprendizaje da lugar al desarrollo de una actitud positiva durante el proceso de enseñanza por parte del alumnado (Lee, Park y Davis, 2018). Expertos como Thai, De Wever y Valcke (2017) han realizado estudios con resultados concordantes con estas líneas, constatando que tanto la motivación como el rendimiento de los discentes que utilizan este enfoque metodológico son mejores que los alcanzados por otros estudiantes que han seguido otras técnicas pedagógicas. Por ello, el aprendizaje invertido se sitúa como un enfoque con un altísimo nivel de eficacia en comparación no solo con otros modelos más clásicos, sino además con otros enfoques de naturaleza innovadora (Sánchez, Jimeno, Pertegal y Mora, 2019).

Por su parte, el aprendizaje invertido permite al alumnado efectuar una planificación y autorregulación del aprendizaje en todas las actividades y tareas desarrolladas (Cerezo, Bernardo, Esteban, Sánchez y Tuero, 2015; Chaves, Trujillo y López, 2016), favoreciendo que este tenga un papel activo y protagonista en su propio proceso de instrucción (Molina, Valenciano y Valencia-Peris, 2015). Todo ello requiere de un gran compromiso, voluntad, actitud positiva y motivación por parte de los estudiantes (Cabero y Llorente, 2015).

En otra línea, la autoestima del discente se considera como un factor relevante para obtener la eficacia deseada en la acción formativa (Esquivel, 2018), logrando la consecución de los diferentes elementos de carácter curricular (Awidi y Paynter, 2019; Cabanach, Souto, Freire y Ferradás, 2015; Nortvig, Petersen y Hattesen, 2018). De igual manera, las prácticas realizadas mediante el aprendizaje invertido logran experimentar una mejora de los resultados obtenidos en las pruebas de evaluación (Karabulut, Jaramillo y Hassall, 2018; Mortensen y Nicholson, 2015; O'Flaherty y Phillips, 2015), haciendo especial resonancia entre el alumnado que normalmente presenta un bajo rendimiento y unas calificaciones —en su mayoría - de carácter negativo (Gross, Pietri, Anderson, Moyano-Camihort y Graham, 2015).

Otra de las potencialidades de este enfoque es la flexibilidad que otorga al proceso de aprendizaje, al estar en contacto con recursos digitales que - del mismo modo- mejoran la participación de los estudiantes (Tourón y Santiago, 2015) en diferentes contextos, tanto en el escolar como en el virtual (Báez y Clunie, 2019), así como un incremento de la actividad grupal (Kwon y Woo, 2017).

El aprendizaje invertido provoca que el alumno —al ser el protagonista de su propia construcción del conocimiento - tenga que tomas las riendas para hacer frente a toda clase de contingencias ocurridas durante el proceso de aprendizaje (Long, Logan y Waugh, 2016). En este sentido, este enfoque propicia que el discente tenga que solucionar problemas que por sí solo no sería capaz, pero sí de manera colaborativa con otros iguales (Bognar, Sablić, y Škugor, 2019).

En última instancia, cabe reseñar la incidencia que tiene el plano familiar y su contexto en todo proceso de enseñanza y aprendizaje. En especial, en el aprendizaje invertido supone un gran aspecto a tener en cuenta, además de la situación laboral del alumnado, cuyo estado va a depender buena parte de la eficacia de tal enfoque innovador. La literatura revela que malas condiciones familiares acarrean deficiencias en el rendimiento académico (Tique et al., 2018). Asimismo, si el estudiantado dispone de una ocupación laboral, esto influirá de manera negativa en su implicación, dedicación y — por consiguiente- rendimiento académico (Carrillo y Ríos, 2013). 
En definitiva, la eficacia en la utilización del aprendizaje invertido va a depender de un análisis previo del profesorado para estudiar en profundidad el contexto de los discentes, con el propósito de llevar a cabo un proceso de individualización del aprendizaje a cada uno de los condicionantes del alumnado, lo que conllevará a una mejor adecuación del modelo al alumno y no al revés (Medranda y Romero, 2018).

\section{Método}

Tras la revisión de la literatura, esta investigación parte de los siguientes objetivos:

- Diseñar y validar un instrumento para valorar la eficacia del aprendizaje invertido en base a condicionantes externos inherentes a la metodología emergente en cuestión.

- Conocer la incidencia de variables exógenas como el contexto familiar, la autoestima, la motivación, la autonomía y variables sociales (sexo, edad y carga laboral) sobre el trabajo desarrollado (calificación, resolución de problemas, trabajo colaborativo y participación) por discentes universitarios a través de una metodología basada en el aprendizaje invertido.

Para lograr el alcance de los objetivos formulados, se ha establecido un diseño experimental de corte descriptivo y correlacional, fundamentado en un método cuantitativo.

\section{Participantes}

Se ha tomado una muestra de naturaleza diacrónica de 360 discentes universitarios con una edad comprendida entre los 18 y 23 años $(M=20$; $\mathrm{DT}=2.03)$. Para su selección, siguiendo las orientaciones de Bisquerra (2004), se ha efectuado un muestreo intencional debido a la facilidad para acceder a la muestra de sujetos. En la tabla 1 se encuentran otras características de los discentes que han participado en esta investigación.

Tabla 1. Muestra de estudio

\begin{tabular}{lccc}
\hline \multirow{2}{*}{ Curso académico } & \multicolumn{2}{c}{ Sexo } & \multirow{2}{*}{ Total } \\
\cline { 2 - 3 } $2015-2016$ & Hombre & Mujer & 134 \\
$2016-2017$ & 48 & 86 & 117 \\
$2017-2018$ & 53 & 64 & 109 \\
\hline Total & 42 & 67 & $\mathbf{3 6 0}$ \\
\hline
\end{tabular}

Fuente: Elaboración propia

\section{Instrumento}

La recogida de datos se ha realizado por medio de un cuestionario de tipo ad hoc (FLEXOQUEST), confeccionado en base al análisis de otras herramientas validadas en sujetos adultos españoles.

El motivo por el cual se optó por diseñar un instrumento específico para obtener los datos se encuentra en lograr que la información recopilada fuera lo más objetiva posible y para ello fue necesario contar con un cuestionario que abarcase los distintos ámbitos de estudio, sin necesidad de emplear diversas herramientas que pudiesen ocasionar sesgos, como la falta de participación e 
implicación de los discentes en un momento de vital relevancia investigadora, como es el proceso de recogida de información.

Las particularidades del cuestionario utilizado se sintetizan en un instrumento compuesto por 46 ítems, clasificados en 5 dimensiones (Social=6 ítems; Motivación=12 ítems; Contexto Familiar $=10$ ítems; Autoestima $=10$ ítems; Autonomía $=8$ ítems) que siguen un formato de respuesta - fundamentalmente - de tipología Likert (de 1-nada a 5-totalmente) y otras de elección cerrada.

El instrumento pasó por un proceso de validación cualitativa a través de un juicio de expertos formado por 14 doctores especialistas en la temática de estudio. Estos expertos brindaron un feedback positivo, no teniendo que llevar a cabo grandes reestructuraciones en el cuestionario. Asimismo, para asegurar la concordancia de las opiniones de los especialistas se efectuó el coeficiente Kappa de Fleiss, obteniendo un $\mathrm{K}=.874$. De igual manera, para concretar la fuerza de concordancia se aplicó la W de Kendall, resultando una W=.886. Estos valores revelan una pertinencia elevada tanto en la similitud de los juicios como en su fuerza de concordancia.

La validación cuantitativa se materializó por medio del análisis de componentes principales (ACP), a través de un análisis factorial exploratorio con rotación de tipo Varimax.

El ACP se efectuó en una muestra —independiente a los sujetos de este estudio- de 323 estudiantes pertenecientes a la Educación Superior. Se obtuvieron 4 factores con eigenvalue mayor que 1. De tal manera que el criterio para asignar el ítem al factor fue la tenencia de una carga factorial $>.3$, demostrado por la varianza total del $67.3 \%$. Del mismo modo, la viabilidad del análisis factorial exploratorio se basó en el $88.9 \%$ de las correlaciones de la matriz que alcanzaron un resultado superior a .3 con un determinante $<.001$. Acto seguido, se halló la prueba de esfericidad de Bartlett (2721.63; $p<.001$ ), hallando una dependencia en las variables de estudio.

Para determinar la adecuación muestral se efectuó el test de Kaiser-Meyer-Olkin, alcanzando un resultado de $\mathrm{KMO}=.945$, dando lugar a que las correlaciones entre pares de variables son esclarecidas por las demás, y por los valores obtenidos en las comunalidades $\left(h^{2}>\right.$.69). De igual forma las medidas de adecuación de la muestra revelaron valores por encima de .85 , reflejando la relevancia de análisis factorial de la matriz de correlaciones.

Sobre la consistencia interna de las cuestiones que conforman el instrumento, el alfa de Cronbach mostró valores superiores a .8 que, siguiendo a expertos como Hernández, Fernández y Baptista (2016), revelan una elevada fiabilidad del mismo.

\section{Procedimiento}

En este estudio se toman los datos relativos a los 3 últimos cursos académicos (20152018). El proceso investigador tiene lugar en 5 fases:

- Primera fase: La primera medida realizada fue conseguir la colaboración del cuerpo docente para desplegar tal enfoque innovador. Para ello, el profesorado tuvo que adaptar sus materiales didácticos, así como la metodología empleada en el proceso de enseñanza y aprendizaje.

- Segunda fase: Una vez obtenida la colaboración docente, se pasó a la recogida del consentimiento de los discentes como sujetos de este estudio, informándoles que los datos obtenidos iban a ser tratados con total anonimato y confidencialidad. 
- Tercera fase: El profesorado — siguiendo las recomendaciones de los investigadoresimpartió los contenidos mediante una metodología de aprendizaje invertido, cuyos vídeos fueron subidos a un edublog, permitiendo el visionado de los contenidos previamente a la sesión presencial y — de tal forma - la interacción y colaboración de los distintos agentes en un espacio digital.

- Cuarta fase: El docente, llegado el momento de evaluar, emplea una rúbrica en la que recoge cada uno de los aspectos de evaluación: a) la calificación obtenida en la prueba teórica; b) el trabajo colaborativo de los estudiantes; c) la participación en las sesiones presenciales y en línea (a través del learning analytics de la plataforma-blog); d) la resolución de problemas prácticos.

- Quinta fase: Aplicación en la muestra de estudiantes del cuestionario validado FLEXOQUEST con el propósito de recoger toda la información requerida para lograr el alcance de los objetivos plasmados en esta investigación.

\section{Análisis de datos}

En el análisis estadístico descriptivo se tomó el número de casos presentes y el porcentaje referido a las variables cualitativas, además de la media (M) y desviación típica (DT) para las variables cuantitativas.

Para determinar la viabilidad del análisis factorial exploratorio se utilizó la prueba de esfericidad de Bartlett, el test de Kaiser-Meyer-Olkin y los valores de las medidas de adecuación muestral.

El proceso de factorización se llevó a cabo por el método de componentes principales con una rotación Varimax, así como el estadístico alfa de Cronbach para revelar el índice de consistencia interna.

Para el análisis confirmatorio del estudio exploratorio efectuado se empleó una ecuación estructural a través del método de extracción de máxima verosimilitud. Asimismo, se realizaron modelos de regresión lineal múltiple para precisar el efecto de las variables independientes (sexo, edad, trabajo, contexto familiar, autoestima, motivación y autonomía) sobre la eficacia (calificación alcanzada, resolución de problemas, trabajo colaborativo y participación de los discentes) del flipped learning.

Para ello, se utilizó el programa IBM Statistical Package for the Social Sciences (SPSS) e IBM SPSS Amos v.25, para Windows, tomando como diferencias estadísticamente significativas aquellas cuya $p<05$.

\section{Resultados}

A continuación se muestran los principales hallazgos obtenidos en este estudio. Tal y como se expone en la figura 1 , se determina la confirmación y adecuación del modelo alcanzado en la fase exploratoria, logrando un modelo compuesto de 4 factores. Los parámetros estimados resultaron estadísticamente significativos, cuyas cargas factoriales se situaron por encima de .57, indicando una saturación de las variables latentes. 


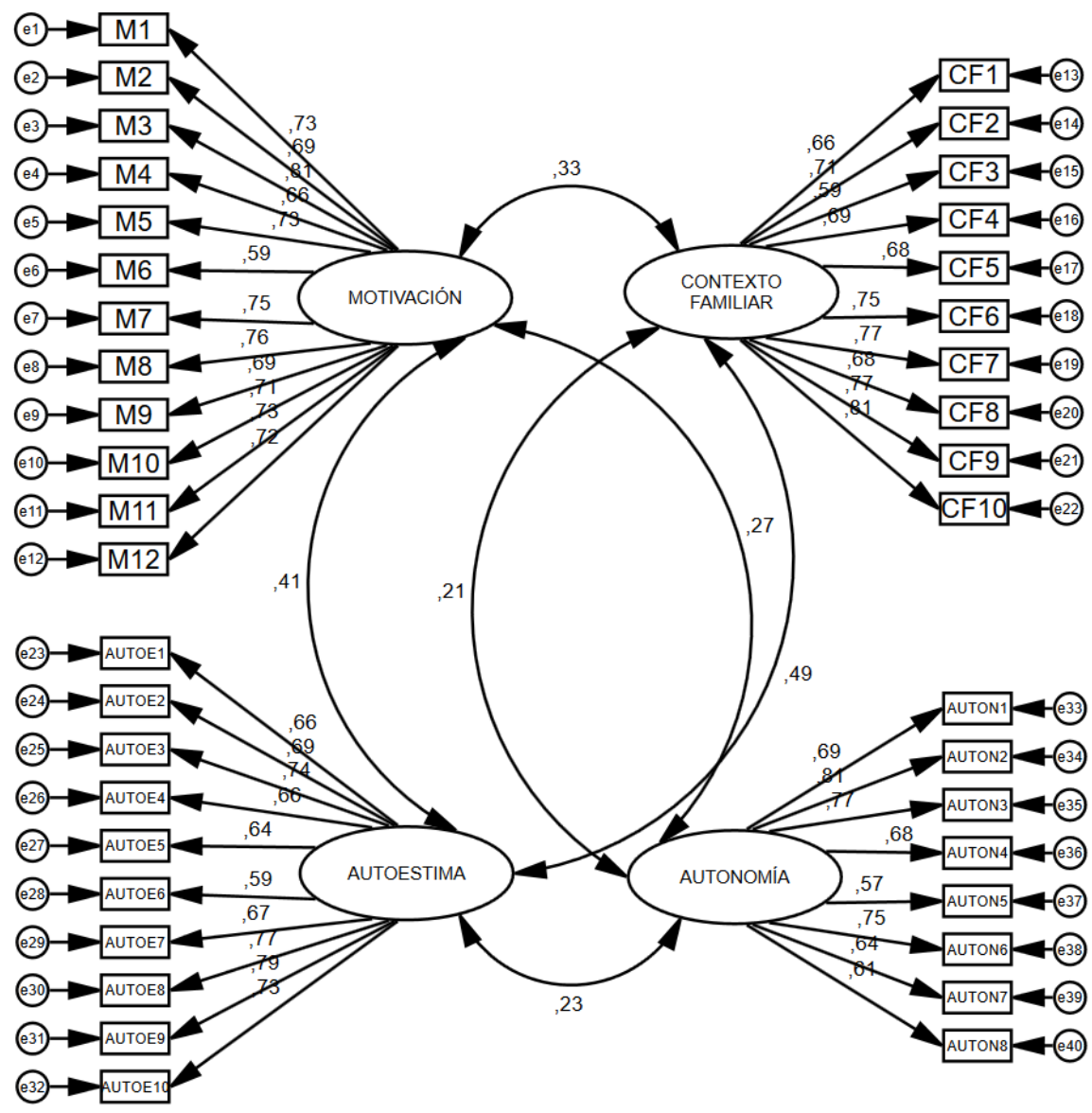

Figura 1. Ecuación estructural del AFC (Elaboración propia)

Los índices de bondad de ajuste (tabla 2) muestran resultados pertinentes, determinando un modelo sustentable sobre la estructura factorial de la escala. Asimismo, se efectuaron - en 2 submuestras de naturaleza independiente y aleatoria - análisis que reportaron valores adecuados en el modelo.

Tabla 2. Índices de bondad de ajuste del AFC

\begin{tabular}{lccccccc}
\hline & $\boldsymbol{\chi} \mathbf{2} / \mathbf{g l}$ & GFI & AGFI & CFI & NFI & TLI & RMSEA (I.C. 90\%) \\
\hline Total & 5.36 & .97 & .96 & .96 & .97 & .96 & $.055(.043-.064)$ \\
Submuestra 1 & 4.43 & .96 & .96 & .95 & .96 & .95 & $.053(.041-.066)$ \\
Submuestra 2 & 4.37 & .95 & .96 & .96 & .95 & .96 & $.054(.044-.069)$ \\
\hline
\end{tabular}

Fuente: Elaboración propia

Una vez aplicado el cuestionario en la muestra de estudio, en la tabla 3 se vierten los valores obtenidos en cada uno de los ítems que componen el instrumento. Se observa que se han alcanzado valores medios en cada uno de los ámbitos concernientes a la investigación desplegada. De entre las variables estudiadas, se aprecia un leve decantamiento de la autonomía del discente sobre el resto de variables tomadas en consideración. 
Tabla 3. Descriptivo de los resultados obtenidos en los diferentes ítems

\begin{tabular}{|c|c|c|}
\hline Ítems & M & DT \\
\hline Motivación & 2.87 & .43 \\
\hline 1. Toma su propio criterio para tomar decisiones. & 2.73 & .41 \\
\hline 2. Prefiere ser parte del grupo de alumnos y ser aceptado por los demás. & 2.41 & .35 \\
\hline 3. Presenta interés por ser superar a los demás. & 2.56 & .48 \\
\hline 4. Persigue la equidad en el ambiente. & 3.02 & .53 \\
\hline 5. Busca superar diferentes retos y alcanzar el éxito. & 2.95 & .42 \\
\hline 6. Dosifica su esfuerzo para conseguir sus propósitos. & 2.64 & .38 \\
\hline 7. Gestiona los resultados de aprendizaje obtenido en las pruebas. & 2.89 & .33 \\
\hline 8. Presenta un estado anímico y una actitud regularizada. & 3.16 & .54 \\
\hline 9. Gusto por los nuevos y variados aspectos del estudio y sus formas de interactuar. & 2.97 & .46 \\
\hline 10. Persigue una situación estable y sin contingencias. & 3.22 & .50 \\
\hline 11. Pretende ofrecer ayuda a los demás, ocasionando un efecto positivo en el entorno. & 3.11 & .44 \\
\hline 12. Protege sus propiedades y a sí mismo. & 2.83 & .37 \\
\hline Contexto Familiar & 2.90 & .45 \\
\hline 1. Presenta ocasiones positivas para el aprendizaje y buenas relaciones con la familia. & 2.54 & .38 \\
\hline 2. Dispone de espacios idóneos que propician la acción formativa. & 3.07 & .46 \\
\hline 3. Presencia de normas en el seno familiar. & 2.81 & .54 \\
\hline 4. Dispone de un contexto familiar de carácter estable. & 2.76 & .42 \\
\hline 5. Los padres presentan algún tipo de antecedentes de índole psicosocial. & 2.93 & .56 \\
\hline 6. Presenta adecuadas condiciones en el hogar y estabilidad económica. & 3.32 & .47 \\
\hline 7. Dispone de determinados apoyos en la familia. & 2.87 & .35 \\
\hline 8. Dispone de un desarrollo familiar adecuado. & 2.96 & .41 \\
\hline 9. Participa y se integra en la comunidad. & 3.03 & .33 \\
\hline 10. Presencia de conflictos dentro del seno familiar. & 2.74 & .57 \\
\hline Autoestima & 2.88 & .48 \\
\hline 1. Se percibe como un buen estudiante. & 2.24 & .44 \\
\hline 2. Se percibe como una persona que desempeña sus labores diarias de manera adecuada. & 2.62 & .53 \\
\hline 3. Presenta facilidad para conocer nuevos amigos o mantener los existentes. & 3.06 & .59 \\
\hline 4. Presenta un adecuado desempeño a la hora de establecer contacto con otras personas. & 2.75 & .47 \\
\hline 5. Presenta un estado emocional adecuado entre sus iguales. & 2.93 & .52 \\
\hline 6. Presenta un estado emocional adecuado con personas de nivel superior. & 3.05 & .59 \\
\hline 7. Se percibe como una persona implicada e integrada en el entorno. & 2.97 & .31 \\
\hline 8. Domina el afecto y la confianza en sí mismo. & 2.92 & .55 \\
\hline 9. Se compromete y se implica en las acciones que lleva a cabo. & 3.15 & .48 \\
\hline 10. Está conforme con su aspecto físico. & 3.08 & .36 \\
\hline Autonomía & 3.02 & .46 \\
\hline 1. Presenta destrezas para comunicarse con los demás. & 3.14 & .57 \\
\hline 2. Presenta destrezas para actuar en el entorno. & 2.96 & .41 \\
\hline 3. Presenta destrezas para lograr ser independiente en labores del día a día. & 3.07 & .52 \\
\hline $\begin{array}{l}\text { 4. Presenta destrezas para cuidar y respetar tanto el hogar familiar como otros lugares en el } \\
\text { que interactúe. }\end{array}$ & 2.88 & .46 \\
\hline 5. Presenta destrezas para autocuidar la salud. & 2.91 & .39 \\
\hline 6. Presenta destrezas relacionadas con la planificación. & 2.98 & .48 \\
\hline 7. Presenta destrezas para elegir entre diferentes alternativas. & 3.09 & .43 \\
\hline 8. Presenta destrezas alusivas a la responsabilidad y el control de sus acciones. & 3.17 & .44 \\
\hline
\end{tabular}

Fuente: Elaboración propia 
La tabla 4 muestra las valoraciones obtenidas en cada uno de los aspectos evaluados que engloba el concepto de eficacia. Tras su análisis, se interpreta que —al igual que las variables anteriormente mostradas - se han reportado resultados muy similares y de corte central en la distribución, siendo el trabajo colaborativo el que se encuentra ligeramente por encima del resto.

Tabla 4. Descriptivo de las puntuaciones alcanzadas en la eficacia

\begin{tabular}{lll}
\hline & Mín.-Máx. & Media (DT) \\
\hline Eficacia & $1-4.75$ & $2.97(.69)$ \\
Calificaciones & $1-5$ & $2.91(1.44)$ \\
Resolución problemas & $1-5$ & $2.99(1.41)$ \\
Trabajo colaborativo & $1-5$ & $3(1.35)$ \\
Participación & $1-5$ & $2.98(1.42)$ \\
\hline
\end{tabular}

Fuente: Elaboración propia

En cuanto al efecto emitido por las variables analizadas sobre la eficacia del aprendizaje invertido, la tabla 5 revela los resultados del modelo de regresión lineal múltiple llevado a cabo. El modelo final resultó ser estadísticamente significativo $[F(7.352)=17.624, p<.001]$, explicando un $49.7 \%$ de la varianza. Las variables socio-demográficas como la edad y la carga laboral arrojaron un efecto significativo en el índice de eficacia de la metodología innovadora. Por tanto, se obtiene que los discentes de mayor edad alcanzan mayores niveles de eficacia. Por el contrario, el desempeño laboral se asocia con niveles bajos de la misma. Sin embargo, el sexo no alcanzó la significancia estadística, revelando que ser hombre o mujer no es determinante para conseguir mayor índice de eficacia en la utilización de tal enfoque innovador.

En lo referido a las variables exógenas, estas revelaron - del mismo modo - un efecto estadísticamente significativo y positivo sobre la eficacia, determinando que la obtención de una elevada eficacia se debe a altos niveles conexos al contexto familiar, autoestima, motivación y autonomía de los estudiantes.

Tabla 5. Efecto producido por las variables socio-demográficas y exógenas en la eficacia

\begin{tabular}{llll}
\hline Variable & $\boldsymbol{B}(\boldsymbol{E T})$ & $\boldsymbol{t}$ & $\boldsymbol{p}$-valor \\
\hline Sexo (Mujer) & $.094(.078)$ & 1.205 & .229 \\
Edad & $.021(.008)$ & 2.625 & .009 \\
Trabajo (Sí) & $-.182(.074)$ & -2.469 & .014 \\
Contexto familiar & $.492(.038)$ & 12.913 & $<.001$ \\
Autoestima & $.071(.032)$ & 2.219 & .027 \\
Motivación & $.081(.037)$ & 2.204 & .028 \\
Autonomía & $.158(.058)$ & 2.715 & .007 \\
\hline
\end{tabular}

Fuente: Elaboración propia

Con respecto a los ámbitos que conforman la eficacia, en las sucesivas tablas se muestran los resultados obtenidos en los modelos de regresión de cada una de ellas. En la calificación 
alcanzada por los estudiantes (tabla 6), se halló un efecto significativo en el desempeño laboral (trabajar se asocia con niveles bajos de eficacia) y en las variables contexto familiar, autoestima y motivación de forma positiva (eminentes niveles se asocian con elevados indicios de eficacia del enfoque innovador).

Tabla 6. Efecto producido por las variables socio-demográficas y exógenas en la calificación

\begin{tabular}{llll}
\hline \multicolumn{4}{l}{ Calificación } \\
\hline Variable & $\boldsymbol{B}(\boldsymbol{E T})$ & $\boldsymbol{t}$ & $\boldsymbol{p}$-valor \\
Sexo (Mujer) & $.08(.16)$ & .46 & .648 \\
Edad & $.04(.05)$ & .81 & .419 \\
Trabajo (Sí) & $-.16(.07)$ & -2.18 & $\mathbf{. 0 3 0}$ \\
Contexto familiar & $.34(.11)$ & 3.12 & $\mathbf{. 0 0 2}$ \\
Autoestima & $.15(.06)$ & 2.52 & $\mathbf{. 0 1 2}$ \\
Motivación & $.06(.03)$ & 2.04 & $\mathbf{. 0 4 2}$ \\
Autonomía & $.20(.15)$ & 1.36 & .174 \\
$\boldsymbol{R}^{2}(\%)$ & 21.9 & & \\
Modelo & $F(7.352)=8.75 ; p<.001$ & \\
\hline
\end{tabular}

Fuente: Elaboración propia

En el modelo de regresión generado para la resolución de problemas por parte de los discentes (tabla 7), las variables que resultaron estadísticamente significativas y de forma positiva fueron la autoestima y la autonomía, determinando que niveles prominentes de éstas se asocian con niveles altos de eficacia de la metodología aprendizaje invertido.

Tabla 7. Efecto producido por las variables socio-demográficas y exógenas en la resolución de problemas

\begin{tabular}{llll}
\hline & \multicolumn{3}{l}{ Resolución de problemas } \\
\hline Variable & $\boldsymbol{B}(\boldsymbol{E}$ T) & $\boldsymbol{t}$ & $\boldsymbol{p}$-valor \\
Sexo (Mujer) & $.04(.16)$ & .22 & .824 \\
Edad & $.05(.04)$ & 1.35 & .177 \\
Trabajo (Sí) & $.03(.16)$ & .16 & .870 \\
Contexto familiar & $.01(.17)$ & .05 & .957 \\
Autoestima & $.57(.16)$ & 3.46 & $\mathbf{. 0 0 1}$ \\
Motivación & $.13(.18)$ & .69 & .492 \\
Autonomía & $.19(.08)$ & 2.48 & $\mathbf{. 0 1 3}$ \\
$\boldsymbol{R}^{\mathbf{2}(\%)}$ & 18.4 & & \\
Modelo & $F(7.352)=3.41 ; p<.001$ & \\
\hline
\end{tabular}

Fuente: Elaboración propia 
Sobre el modelo de regresión establecido para el trabajo colaborativo desplegado por los alumnos (tabla 8), las variables que resultaron estadísticamente significativas y de forma positiva - al igual que en la resolución de problemas - fueron la autoestima y la autonomía, revelando que insignes niveles de éstas se vinculan con índices elevados de eficacia de la metodología emergente aplicada.

Tabla 8. Efecto producido por las variables socio-demográficas y exógenas en el trabajo colaborativo

\begin{tabular}{llll}
\hline & \multicolumn{3}{l}{ Trabajo colaborativo } \\
\hline Variable & $\boldsymbol{B}(\boldsymbol{E T})$ & $\boldsymbol{t}$ & $\boldsymbol{p}$-valor \\
Sexo (Mujer) & $.07(.06)$ & 1.34 & .182 \\
Edad & $.02(.04)$ & .57 & .568 \\
Trabajo (Sí) & $.02(.15)$ & .11 & .913 \\
Contexto familiar & $.10(.16)$ & .64 & .526 \\
Autoestima & $.23(.05)$ & 4.93 & $<.001$ \\
Motivación & $.01(.17)$ & .03 & .977 \\
Autonomía & $.15(.07)$ & 2.06 & $\mathbf{. 0 4 0}$ \\
$\boldsymbol{R}^{2}(\%)$ & 17.9 & & \\
Modelo & $F(7.352)=4.48 ; p<.001$ & \\
\hline
\end{tabular}

Fuente: Elaboración propia

En lo concerniente a la participación (tabla 9), en el modelo de regresión efectuado resultaron significativas y de forma positiva la autoestima y la motivación, por lo que se verifica que niveles altos en ellas se relacionan con niveles sobresalientes de eficacia en el aprendizaje invertido.

Tabla 9. Efecto producido por las variables socio-demográficas y exógenas en la participación

\begin{tabular}{llll}
\hline & \multicolumn{2}{l}{ Participación } & \\
\hline Variable & $\boldsymbol{B}(\boldsymbol{E T})$ & $\boldsymbol{t}$ & $\boldsymbol{p}$-valor \\
Sexo (Mujer) & $.16(.10)$ & 1.57 & .116 \\
Edad & $.05(.04)$ & 1.35 & .177 \\
Trabajo (Sí) & $.15(.16)$ & .93 & .352 \\
Contexto familiar & $.02(.17)$ & .14 & .886 \\
Autoestima & $.27(.07)$ & 3.75 & $<.001$ \\
Motivación & $.14(.06)$ & 2.14 & $\mathbf{. 0 3 3}$ \\
Autonomía & $.20(.15)$ & 1.34 & .179 \\
$\boldsymbol{R}^{\mathbf{2}}$ \%) & 17.7 & & \\
Modelo & $F(7.352)=4.90 ; p<.001$ & \\
\hline
\end{tabular}

Fuente: Elaboración propia 


\section{Discusión y conclusiones}

Como se muestra de manera constatada a través de la revisión de la literatura sobre los estudios realizados de mayor impacto en torno al aprendizaje invertido — como metodología innovadora en un mundo en el que está de moda el aprendizaje a través de entornos digitales - se ha comprobado a través de un análisis de contenido que esta forma de enseñar y aprender aumenta la efectividad del aprendizaje (He et al., 2016; Sánchez et al., 2019), ayuda a promover la motivación de los discentes (Shih y Tsai, 2017; Thai et al., 2017; Tse et al., 2019), aumenta de forma significativa la participación de estos en las situaciones de aprendizaje formal (Long et al., 2016) e incluso la capacidad para resolver problemas o situaciones planteadas en clase (Bognar et al., 2019; Long et al., 2016). Los estudiantes trabajarán así colaborando entre todos para construir su aprendizaje, de manera cooperativa para poder alcanzar las metas planteadas (Pegalajar, 2018), tanto de manera física o presencial como a través de entornos virtuales (Báez y Clunie, 2019; Kwon y Woo, 2017; MacLeod et al., 2017).

Todos estos factores contribuyen a la mejora de las calificaciones de los estudiantes (Awidi y Paynter, 2019; Karabulut et al., 2018; Mortensen y Nicholson, 2015; Nortvig et al., 2018; O'Flaherty y Phillips, 2015). Pero no solamente se ven aumentadas las notas de los estudiantes - aparte de todos los beneficios anteriormente mencionados - sino que con el uso de esta metodología se potencia también la autonomía (Chaves et al., 2016).

En base a los hallazgos alcanzados en la presente investigación, se ha podido constatar la confección y validación de un instrumento para la medición de la influencia de variables extrínsecas conexas al aprendizaje invertido.

Igualmente, al aplicar la herramienta generada, tanto en los factores exógenos atribuidos al aprendizaje invertido, como en los constructos delimitados para valorar la eficacia de la metodología innovadora, se han obtenidos cifras centrales y muy similares, destacando sutilmente las puntuaciones referidas a la autonomía manifestada por los discentes y el trabajo colaborativo desplegado por estos.

En este estudio se ha podido concluir que el sexo de los estudiantes no influye a la hora de conseguir un aprendizaje más eficaz mediante la metodología de aprendizaje invertido, por lo que ser hombre o mujer no es un factor decisivo para alcanzar el éxito en los resultados. Aunque sí influyen variables como la edad y la situación laboral de los mismos, comprobándose que los estudiantes de edad superior han conseguido mayores índices de eficacia sobre el resto. Así como, los estudiantes que trabajan a la vez que estudian revelan menores índices de la misma.

Sobre los aspectos que conforman la eficacia (calificación, resolución de problemas, trabajo colaborativo y participación del alumnado), la realización de este estudio ha permitido extraer que factores externos ejercen mayor influencia sobre cada uno de los ámbitos que se han tomado para alcanzar la eficacia del modelo. En lo concerniente a la calificación obtenida por los estudiantes, son el contexto familiar, la autoestima y la motivación las que ejercen una mayor incidencia. De igual forma, tanto la resolución de problemas como el trabajo colaborativo, son la autoestima y la autonomía los factores más determinantes. Y — por último - en la participación se ha concretado que la autoestima y la motivación son los factores extrínsecos más influyentes.

Con respecto a los factores externos de esta metodología (establecidos en esta investigación) se puede concluir que los estudiantes que tienen un contexto familiar adecuado y 
niveles aceptables de autonomía, motivación y autoestima han conseguido mayores índices de eficacia en el aprendizaje.

Esta metodología emergente es considerada como uno de los actuales enfoques innovadores, ejerciendo el alumno un papel activo (Berenguer, 2016) para construir su aprendizaje, pero esto también requiere un doble esfuerzo previo por parte del docente, que tiene que realizar o preparar los materiales audiovisuales de forma previa a las clases presenciales, significando un aumento de la carga de trabajo habitual, aspecto que podría considerarse como una limitación para muchos docentes.

Como futura línea de investigación se propone analizar la incidencia de los factores extrínsecos - tenidos en cuenta en este estudio - en otras etapas educativas como la Educación Secundaria Obligatoria y el Bachillerato.

\section{Referencias}

Area, M., Hernández, V. y Sosa, J. J. (2016). Modelos de integración didáctica de las TIC en el aula. Comunicar: Revista científica iberoamericana de comunicación y educación, 24(47), 79-87. DOI: 10.3916/C47-2016-08

Awidi, I. T. y Paynter, M. (2019). The impact of a flipped classroom approach on student learning experience. Computers \& Education, 128, 269-283.

Báez, C. I. y Clunie, C. E. (2019). Una mirada a la Educación Ubicua. RIED. Revista Iberoamericana de Educación a Distancia, 22(1), 325-344. DOI: 10.5944/ried.22.1.22422

Berenguer, C. (2016). Acerca de la utilidad del aula invertida o flipped classroom. En M. Tortosa, S. Grau y J. Álvarez (Ed.), XIV Jornadas de redes de investigación en docencia universitaria. Investigación, innovación y enseñanza universitaria: enfoques pluridisciplinares. (pp. 1466- 1480). Alicante, España: Universitat d'Alacant.

Bisquerra, R. (2004). Metodología de la investigación educativa. Madrid: La Muralla.

Boelens, R., Voet, M. y De Wever, B. (2018). The design of blended learning in response to student diversity in higher education: Instructors' views and use of differentiated instruction in blended learning. Computers \& Education, 120, 197-212. DOI: 10.1016/j.compedu.2018.02.009

Bognar, B., Sablić, M. y Škugor, A. (2019). Flipped Learning and Online Discussion in Higher Education Teaching. En C. Reidsema, L. Kavanagh, R. Hadgraft y N. Smith (Eds.), The flipped classroom: Practice and practices in higher education (pp. 371-392). Nueva York, EE. UU.: Springer. DOI: 10.1007/978-3-030-01551-0_19

Cabanach, R. G., Souto, A., Freire, C. F. y Ferradás, M. M. (2015). Relaciones entre autoestima y estresores percibidos en estudiantes universitarios. European Journal of Education and Psychology, 7(1), 43-57. DOI: 10.1989/ejep.v7i1.151

Cabero, J. y Llorente, M. C. (2015). Tecnologías de la Información y la Comunicación (TIC): escenarios formativos y teorías del aprendizaje. Revista Lasallista de Investigación, 12(2), 186-193. Recuperado de: http://www.redalyc.org/articulo.oa?id=69542291019

Carrillo, S., y Ríos, J. G. (2013). Trabajo y rendimiento escolar de los estudiantes universitarios. El caso de la Universidad de Guadalajara, México. Revista de la educación superior, 42(166), 9-34. Recuperado de http://www.scielo.org.mx/scielo.php?script=sci_arttext\&pid=S0185$27602013000200001 \& \operatorname{lng}=$ es\&tlng $=$ es 
Cerezo, R., Bernardo, A., Esteban, M., Sánchez, M. y Tuero, E. (2015). Programas para la promoción de la autorregulación en educación superior: un estudio de la satisfacción diferencial entre metodología presencial y virtual. European Journal of Education and Psychology, 8(1), 30-36. Recuperado de: https://www.sciencedirect.com/science/article/pii/S1888899215000112

Chaves, E., Trujillo, J. M. y López, J. A. (2016). Acciones para la autorregulación del aprendizaje en entornos personales de aprendizaje. Pixel-bit: revista de medios $y$ educación, 48, 67-82. DOI: 10.12795/pixelbit.2016.148.05

El Miedany, Y. (2019). Flipped Learning. En C. Reidsema, L. Kavanagh, R. Hadgraft y N. Smith (Eds.), The flipped classroom: Practice and practices in higher education (pp. 285-303). Nueva York, EE. UU.: Springer. DOI: 10.1007/978-3-319-98213-7_15

Esquivel, J. C. (2018). Autoestima y su relación con el rendimiento académico en estudiantes del sistema de universidad abierta. Facultad de Educación. Universidad Católica los Ángeles de Chimbote-Huaraz, 2015. Aporte Santiaguino, 11(1), 157-166. DOI: 10.32911/as.2018.v11.n1.464

Fernández, F. J., Fernández M. J. y Rodríguez, J. M. (2018). El proceso de integración y uso pedagógico de las TIC en los centros educativos madrileños. Educación XX1, 21(2), 395416. DOI: $10.5944 /$ educXX1.17907

Froehlich, D. E. (2018). Non-technological learning environments in a technological world: Flipping comes to the aid. Journal of New Approaches in Educational Research, 7(2), 9499. DOI: 10.7821/naer.2018.7.304

Gross, D., Pietri, E. S., Anderson, G., Moyano-Camihort, K. y Graham, M. J. (2015). Increased preclass preparation underlies student outcome improvement in the flipped classroom. CBE_Life Sciences Education, 14(4), 1-8. DOI: 10.1187/cbe.15-02-0040

He, W., Holton, A., Farkas, G. y Warschauer, M. (2016). The effects of flipped instruction on out-of-class study time, exam performance, and student perceptions. Learning and Instruction, 45, 61-71. DOI: 10.1016/j.learninstruc.2016.07.001

Hernández, R., Fernández, C. y Baptista, M. P. (2016). Metodología de la investigación. México: McGraw Hill.

Jovanović, J., Gašević, D., Dawson, S., Pardo, A. y Mirriahi, N. (2017). Learning analytics to unveil learning strategies in a flipped classroom. The Internet and Higher Education, 33(4), 74-85. DOI: 10.1016/j.iheduc.2017.02.001

Karabulut, A., Jaramillo, N. y Hassall, L. (2018). Flipping to engage students: Instructor perspectives on flipping large enrolment courses. Australasian Journal of Educational Technology, 34(4), 123-137. DOI: 10.14742/ajet.4036

Kwon, J. E. y Woo, H. R. (2017). The Impact of Flipped Learning on Cooperative and Competitive Mindsets. Sustainability, 10(79), 1-15. DOI: 10.3390/su10010079

Lee, J., Lim, C. y Kim, H. (2017). Development of an instructional design model for flipped learning in higher education. Educational Technology Research and Development, 65(2), 427-453. DOI 10.1007/s11423-016-9502-1

Lee, J., Park, T. y Davis, R. O. (2018). What affects learner engagement in flipped learning and what predicts its outcomes?. British Journal of Educational Technology. 1(1), 1-18. DOI: $10.1111 /$ bjet.12717 
Long, T., Cummins, J. y Waugh, M. (2017). Use of the flipped classroom instructional model in higher education: instructors' perspectives. Journal of Computing in Higher Education, 29(2), 179-200. DOI 10.1007/s12528-016-9119-8

Long, T., Logan, J. y Waugh, M. (2016). Students' perceptions of the value of using videos as a pre-class learning experience in the flipped classroom. TechTrends, 60(3), 245-252. DOI: $10.1007 / \mathrm{s} 11528-016-0045-4$

López, M. y Bernal, C. (2019). El perfil del profesorado en la Sociedad Red: reflexiones sobre la competencia digital de los y las estudiantes en Educación de la Universidad de Cádiz. IJERI: International Journal of Educational Research and Innovation, (11), 83-100. Recuperado de https://www.upo.es/revistas/index.php/IJERI/article/download/3265/2955

MacLeod, J., Yang, H. H., Zhu, S. y Shi, Y. (2017). Technological factors and student-to-student connected classroom climate in cloud classrooms. Journal of Educational Computing Research, 56(6), 826-847. DOI: 10.1177/0735633117733999

McCarthy, J. (2016). Reflections on a flipped classroom in first year higher education. Issues in Educational Research, 26(2), 332-350. Recuperado de http://www.iier.org.au/iier26/mccarthy-j.pdf

Medranda, R. F. y Romero, S. (2018). Problemas familiares y rendimiento académico. Revista Caribeña de Ciencias Sociales, 4(2), 1-11. Recuperado de https://www.eumed.net/rev/caribe/2018/05/estudiantes-trabajo-social.html

Molina, P., Valenciano, J., y Valencia-Peris, A. (2015). Los blogs como entornos virtuales de enseñanza y aprendizaje en Educación Superior. Revista Complutense de Educación, 26, 15-31. DOI: http://dx.doi.org/10.5209/rev_RCED.2015.v26.43791

Mortensen, C. J. y Nicholson, A. M. (2015). The flipped classroom stimulates greater learning and is a modern 21 st century approach to teaching today's undergraduates. Journal of animal science, 93(7), 3722-3731. DOI: 10.2527/jas.2015-9087

Nortvig, A. M., Petersen, A. K. y Hattesen, S. (2018). A Literature Review of the Factors Influencing E-Learning and Blended Learning in Relation to Learning Outcome, Student Satisfaction and Engagement. Electronic Journal of e-Learning, 16(1), 46-55. Recuperado de https://files.eric.ed.gov/fulltext/EJ1175336.pdf

O'Flaherty, J. y Phillips, C. (2015). The use of flipped classrooms in higher education: A scoping review. The internet and higher education, 25, 85-95. DOI: 10.1016/j.iheduc.2015.02.002

Pegalajar, M. C. (2018). Formación en competencias en alumnado universitario de Educación Social mediante prácticas basadas en aprendizaje cooperativo. Revista Complutense de Educación, 29(3), 829-845. http://dx.doi.org/10.5209/RCED.53970

Pereira, S., Fillol, J. y Moura, P. (2019). El aprendizaje de los jóvenes con medios digitales fuera de la escuela: De lo informal a lo formal. Comunicar: Revista científica iberoamericana de comunicación y educación, 27(58), 41-50. DOI: 10.3916/C58-2019-04

Rotellar, C. y Cain, J. (2016). Research, perspectives, and recommendations on implementing the flipped classroom. American journal of pharmaceutical education, 80(2), 1-9. DOI: 10.5688/ajpe 80234 
Sánchez, C. (2017). Flipped classroom. La clase invertida, una realidad en la Facultad de Ciencias de la Educación de la Universidad de Málaga (Tesis doctoral). Universidad de Málaga, España. Recuperado de https://riuma.uma.es/xmlui/bitstream/handle/10630/14993/TD_SANCHEZ_CRUZADO_ Cristina.pdf? sequence $=1$ \&isAllowed $=\mathrm{y}$

Sánchez, J. L., Jimeno, A., Pertegal, M. L. y Mora, H. (2019). Design and application of Projectbased Learning Methodologies for small groups within Computer Fundamentals subjects. IEEE Access, 7, 12456-12466. DOI: 10.1109/ACCESS.2019.2893972

Seery, M. K. (2015). Flipped learning in higher education chemistry: emerging trends and potential directions. Chemistry Education Research and Practice, 16(4), 758-768. DOI: 10.1039/c5rp00136f

Shih, W. L. y Tsai, C. Y. (2017). Students' perception of a flipped classroom approach to facilitating online project-based learning in marketing research courses. Australasian Journal of Educational Technology, 33(5), 32-49. DOI: 10.14742/ajet.2884

Thai, N. T. T., De Wever, B. y Valcke, M. (2017). The impact of a flipped classroom design on learning performance in higher education: Looking for the best "blend" of lectures and guiding questions with feedback. Computers \& Education, 107, 113-126. DOI: 10.1016/j.compedu.2017.01.003

Tique, M. A., Camacho, J. A., Segura, Y. C., Orozco, R., Ortega, L. L., Iriarte, M. ... Cáceres, N. A. (2018). Contexto socioeconómico sobre el rendimiento académico del estudiante mediante la investigación como estrategia pedagógica. Cultura Educación y Sociedad, 9(1), 171-180. DOI: 10.17981/cultedusoc.9.1.2018.13

Tourón, J. y Santiago, R. (2015). El modelo Flipped Learning y el desarrollo del talento en la escuela $=$ Flilpped Learning model and the development of talent at school. Revista de educación, (368), 174-195. DOI: 10.4438/1988-592X-RE-2015-368-288

Tse, W. S., Choi, L. Y. y Tang, W. S. (2019). Effects of video-based flipped class instruction on subject reading motivation. British Journal of Educational Technology, 50(1), 385-398. DOI:10.1111/bjet.12569

Zainuddin, Z., Habiburrahim, H., Muluk, S. y Keumala, C. M. (2019). How do students become self-directed learners in the EFL flipped-class pedagogy? A study in higher education. Indonesian Journal of Applied Linguistics, 8(3). DOI: 10.17509/ijal.v8i3.15270 